\title{
Patterning back to front
}

In theory, patterning the Drosophila embryo should be simple: morphogen gradients that are distributed across the unpatterned tissue activate a combination of transcription factors that interact to define the boundaries of the adult anatomical structures. Of course, working out exactly how combinations of transcription factors delineate borders of gene expression is rather more complicated. Two recent papers focus on the interplay between such factors in the dorsoventral patterning of the fly embryo; their results indicate that distinct thresholds of response to morphogen activity are shaped by the simple balance of positive and negative transcriptional inputs. An activity gradient of Decapentaplegic (Dpp), the homologue of vertebrate TGF- $\beta$, is crucial to the patterning of the dorsoventral axis of the fly embryo. Specifically, Dpp signalling gradients pattern the dorsal ectoderm, with Dpp levels highest at the dorsal midline of the embryo, and gradually declining towards the dorsolateral and lateral domains.
The expression of the tolloid gene is induced at low levels of Dpp throughout the dorsal ectoderm. By dissecting the elements that are required for its expression, Zhang $e t$ al. discovered that Tolloid is restricted to the dorsal ectoderm by the direct action of Brinker (Brk), a sequence-specific transcriptional repressor. Either alone, or in a complex with the co-repressor Groucho, Brk helps to promote neurogenesis by blocking Dpp signalling in the ventral domain of the embryo.

Brk is also the protagonist of the second study, by Rushlow et al., which focused on the expression of zerknüllt (zen), another Dpptarget gene. The restriction of zen expression to the dorsal domain of the embryo requires the positive input of the Dpp-transducer Mad, and the negative effect of Brk. The latter prevents the expansion of zen into more ventral regions. At the molecular level, Brk and Mad compete for binding to their overlapping consensus sites in the zen promoter, suggesting that competition between the two transcriptional regulators sets spatial thresholds for the expression of Dpp-target genes. The later refinement of zen expression to the dorsal-most cells depends on much higher levels of Mad, but becomes independent of Brk.

In principle, the concentration-dependent response to a morphogen such as Dpp could be explained in one of two ways: by activating target gene promoters that respond to different concentrations of transcriptional activators, or by modulating the activity of a negative regulator. The latter explanation seems to hold sway in the examples of zen and tolloid described here.

The promoter profiles of these two genes should enable us to predict the organization of regulatory elements in other Dpp response elements and, more broadly, to test the validity of the competitive binding model in other developmental contexts.

Tanita Casci

\section{(0) References and links}

ORIGINAL RESEARCH PAPERS Zhang, H. et al. Brinker is a sequence-specific transcriptional repressor in the Drosophila embryo. Genes Dev. 15, 261-266 (2001) | Rushlow, C. et al. Transcriptional regulation of the Drosophila gene zen by competing Smad and Brinker inputs. Genes Dev. 15, 340-351 (2001)

ENCYCLOPEDIA OF LIFE SCIENCES Drosophila embryo: dorsal-ventral specification

WEB SITE Christine Rushlow's lab

\section{Tiled arrays for gene hunting}

Guessing the number of human genes is a speculative enterprise. Even with the publication of the draft human genome sequence (see pages 158-159), one can only have cautious confidence in the estimated count of 30,000-40,000. More accurate estimates require a highthroughput method for validation of gene predictions, and Mark Boguski and colleagues now report an approach to do just that using microarray technology.

Traditional computational methods for predicting gene number integrate information from numerous sources, such as sequence signatures of gene structure, similarity to genes in other organisms and evidence that a DNA sequence is expressed. Although collectively powerful, each type of information is subject to error. Furthermore, computational approaches overlook the complexity of gene expression, such as the tremendous diversity that arises from alternative splicing of gene transcripts.

The premise of Boguski and colleagues is that exons of the same gene will demonstrate similar expression patterns across a range of different cell types and experimental conditions. To test this, they designed "exon arrays", comprising 60-mer oligonucleotide probes each of which corresponds to a predicted exon - printed on glass slides. As proof of principle, the authors analysed gene predictions for chromosome 22, the first chromosome to be fully sequenced and exhaustively annotated. Although the method validated most known genes on chromosome 22 , about $15 \%$ were missed, indicating that the method needs further refinement. Intriguingly, over half of the gene predictions based solely on ab initio computer predictions were confirmed - far exceeding earlier expectations - illustrating that this is an effective means of quickly assessing the validity of computational predictions.

To define gene structure more accurately, Boguski and colleagues used higher-resolution 'tiling arrays', in which 60-mer probes are tiled at 10-base-pair intervals across a genomic region of interest. The tiling approach enabled the gene structure of a novel testis transcript to be refined, clarifying the exact exon-intron boundaries and precise transcript length.

As an initial step towards whole genome analysis, the authors constructed 50 arrays that contained over one million probes representing more than 400,000 predicted human exons. The authors detected $58 \%$ of confirmed exons and $34 \%$ of predicted exons (from the Ensembl human genome annotation data set), but only two cell lines were used for the analysis. Although a long way from comprehensively defining the structure of every gene in the human genome, this new approach offers a rapid means of validating computational predictions and training the next generation of genehunting programs.

Carina Dennis, Senior Editor, Nature

\section{(D) References and links}

ORIGINAL RESEARCH PAPER Shoemaker, D. D. et al.

Experimental annotation of the human genome using microarray technology. Nature 409, 922-927 (2001)

WEB SITE Rosetta Inpharmatics

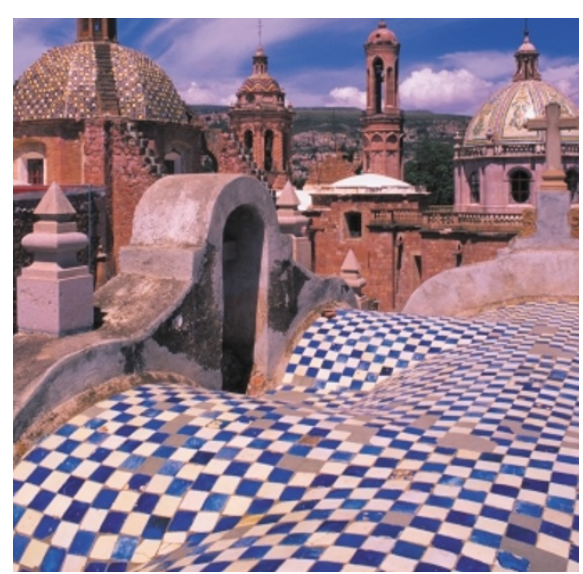

